\title{
Spin-dependent tunneling in diluted magnetic semiconductor trilayer structures
}

\author{
P. Krstajić* and F. M. Peeters ${ }^{\dagger}$ \\ Departement Fysica, Universiteit Antwerpen, Groenenborgerlaan 171, B-2020 Antwerpen, Belgium
}

(Received 3 June 2005; revised manuscript received 2 August 2005; published 30 September 2005)

\begin{abstract}
Tunneling of holes through a trilayer structure made of two diluted magnetic semiconductors, $(\mathrm{Ga}, \mathrm{Mn}) \mathrm{As}$, separated by a thin layer of nonmagnetic AlAs is investigated. The problem is treated within the $6 \times 6$ Luttinger-Kohn model for valence bands with the split-off band included. The influence of the spin-orbit coupling is pronounced as the spin-splitting $\Delta_{e x}$ is comparable with the split-off $\Delta_{S O}$ splitting. It is assumed that direct tunneling is the dominant mechanism due to the high quality of the tunnel junctions. Our theoretical results predict the correct order of magnitude for the tunneling magnetoresistance ratio, but various other effects, such as scattering on impurities and defects, should be included in order to realize a quantitative agreement with experiment.
\end{abstract}

DOI: 10.1103/PhysRevB.72.125350

PACS number(s): 75.50.Pp, 73.40.Kp, 73.50.Jt

\section{INTRODUCTION}

There has been continuing interest in the spin-dependent tunneling in diluted magnetic semiconductors (DMS), due to its possible application in devices such as sensitive read heads ${ }^{1}$ in data storage systems, spin transistors, ${ }^{2}$ magnetic memories, ${ }^{3}$ etc. Giant magnetoresistance (GMR) has been realized in a form of a spin valve and gained acceptance in commercial hard disks during the past 5-10 years. Due to technological reasons, the materials used are (semi)metallic ferromagnets and conductors. The modulation of the resistance during reading of a magnetic disk, i.e., $[R(H)-R(0)] / R(0)$, in these types of heads is typically ${ }^{1}$ $20 \%$. A promising alternative is to use magnetic tunneling junction (MTJ) devices that consist, at their core, of two thin ferromagnetic layers separated by a semiconductor, where tunneling magnetoresistance can reach 40-50\%. With the advent of DMS materials like ( $\mathrm{Ga}, \mathrm{Mn}) \mathrm{As}$, several groups have achieved higher TMR, in trilayer structures made of two (Ga,Mn)As separated by AlAs (barrier) up to $290 \%$ (Ref. 4) and 75\%.5, However, the low Curie temperature (below room temperature) of many III-V DMS still poses a serious obstacle to its wide commercial use. In this paper we concentrate on the theoretical investigation of hole tunneling through a (Ga,Mn)As/GaAs/AlAs/GaAs/(Ga, Mn)As structure, where a thin layer of GaAs is used in experimental setups to avoid diffusion ${ }^{7}$ of $\mathrm{Mn}$ into AlAs, avoid unwanted strain, and make interfaces smoother. On the other hand, tunneling processes and spin polarization have already been treated $^{8}$ in double barrier structures made of (Ga, Mn)As/GaAs within the single band model. However, a more detailed $6 \times 6$ Luttinger-Kohn ${ }^{9}$ Hamiltonian with the exchange included ${ }^{10-12}$ has been employed to estimate theoretically the value of GMR in similar structures. A similar theoretical framework was already used previously to investigate charge and spin distribution in $(\mathrm{Ga}, \mathrm{Mn}) \mathrm{As} / \mathrm{GaAs}$ multilayers. ${ }^{13}$ Furthermore, the success of $\mathbf{k} \cdot \mathbf{p}$ theory to de- termine resonant peaks ${ }^{14}$ and tunneling lifetimes in double barrier structures ${ }^{15}$ gives us confidence to apply it to ferromagnetic DMS trilayers as well, as several authors have already done in other structures. ${ }^{11,16}$

The paper is organized as follows. In Sec. II the $6 \times 6$ Luttinger-Kohn (LK) model with $p$ - $d$ exchange is presented, with particular attention paid to its application to trilayer structures. In Sec. III we give theoretical results for the tunneling magnetoresistance, and discuss its agreement with available experimental data. In Sec. IV, results are compared with a single band model approximation, where band mixing is neglected. A summary of the results is given in Sec. V.

\section{MODEL}

The $6 \times 6$ Luttinger-Kohn model is an effective mass model for holes, where only the basic idea of the cubic symmetry of semiconductor crystal is utilized, while major (Luttinger) parameters are fitted according to empirical data. Holes in III-V semiconductors have angular momentum $l$ $=1$, so that, in the absence of external fields, there are three doubly degenerate bands: heavy hole ( $\mathrm{HH})$ band, light hole (LH) band, and split-off (SO) band. While in nonmagnetic GaAs/AlAs heterostructures it is still possible to neglect the SO band $\left(\epsilon_{F} \gg \Delta_{S O}\right)$ and use the $4 \times 4 \mathrm{LK},{ }^{17}$ the inclusion of all three bands is necessary in DMS since the spin-splitting of holes is comparable with $\Delta_{S O}$. The Hamiltonian with exchange reads

$$
H=H_{6 \times 6}+V(z) I_{6}+\Delta_{e x} s_{x}
$$

The $H_{6 \times 6}$ is a $6 \times 6$ Luttinger-Kohn Hamiltonian, 


$$
H_{6 \times 6}=\left(\begin{array}{cccccc}
P_{1} & L & M & 0 & i L / \sqrt{2} & -i \sqrt{2} M \\
L^{\dagger} & P_{2} & 0 & M & -i \sqrt{2} Q & i \sqrt{3 / 2} L \\
M^{\dagger} & 0 & P_{2} & -L & -i \sqrt{3 / 2} L & -i \sqrt{2} Q \\
0 & M^{\dagger} & -L^{\dagger} & P_{1} & -i \sqrt{2} M^{\dagger} & -i L^{\dagger} / \sqrt{2} \\
-i L^{\dagger} / \sqrt{2} & i \sqrt{2} Q & i \sqrt{3 / 2} L & i \sqrt{2} M & P+\Delta_{S O} & 0 \\
+i \sqrt{2} M^{\dagger} & -i \sqrt{3 / 2} L^{\dagger} & i \sqrt{2} Q & i L \sqrt{2} & 0 & P-\Delta_{S O}
\end{array}\right),
$$

where the matrix elements of the Hamiltonian are given by

$$
\begin{gathered}
P=\frac{\hbar^{2}}{2 m_{0}} \gamma_{1} k^{2}, \quad Q=\frac{\hbar^{2}}{2 m_{0}} \gamma_{2}\left(k^{2}-2 k_{z}^{2}\right), \\
P_{1}=P+Q \quad P_{2}=P-Q, \\
L=-i \frac{\sqrt{3} \hbar^{2}}{m_{0}} \gamma_{3}\left(k_{x}-i k_{y}\right) k_{z}, \\
M=\frac{\sqrt{3} \hbar^{2}}{2 m_{0}}\left(\gamma_{2}\left(k_{x}^{2}-k_{y}^{2}\right)-2 i \gamma_{3} k_{x} k_{y}\right),
\end{gathered}
$$

while $\Delta_{e x}$ is the exchange splitting at $\mathbf{k}=0$ between the two heavy hole states, and $s_{x}$ is the operator of hole spins in the $|j, m\rangle$ basis. The quantization axis is commonly chosen to be along the growth direction $z$. On the other hand, it is known that the easy magnetization of ferromagnetic layers of (Ga,Mn)As is in plane, when grown on GaAs substrate due to the compressive strain. Note that the lattice constant of (Ga,Mn)As increases with Mn content. In the present model the $p$ - $d$ exchange between holes and $d$ electrons on Mn, parametrized by the $\beta$ integral, is taken in the mean-field approximation. The value of $\Delta_{e x}$ can be estimated to be $x S(T) \beta N_{0}$, where $S(T)$ is roughly given by the Brillouin function, and $N_{0}$ is the number of cations per unit area. If the magnetization $M$ of the sample is experimentally known, then a better estimate would be $\Delta_{e x}=\beta M /\left(g \mu_{B}\right)$. The internal magnetic field $\left(B_{\text {int }}=\mu_{0} M\right)$ is considered to have a negligible effect on the orbital motion of the holes ( $k_{\alpha}$ are unchanged), and also the ordinary Zeeman effect is omitted from the Hamiltonian. Here $\gamma_{i}$ are the well-known Luttinger parameters fitted according to experimental data. The basis eigenfunctions $\{|j, m\rangle\}(j=3 / 2)$ are ordered as $\quad\{|3 / 2,3 / 2\rangle,|3 / 2,1 / 2\rangle,|3 / 2,-1 / 2\rangle,|3 / 2,-3 / 2\rangle$, $|1 / 2,1 / 2\rangle|1 / 2,-1 / 2\rangle\}$ and represent the periodic part of the Bloch functions at $\mathbf{k}=\mathbf{0}\left(\Gamma_{8}\right)$. In each $n$th layer the wave function has the following form:

$$
\Psi(\mathbf{r})=e^{i\left(k_{x} x+k_{y} y\right)} \psi(z),
$$

with

$$
\psi(z)=\sum_{i=1, n_{b}}\left[A_{n, i}^{+} V_{n, i}^{+} e^{i k_{n, i}^{+} z}+A_{n, i}^{-} V_{n, i}^{-} e^{i k_{n, i^{-}}^{-}}\right] .
$$

Here $V_{i}$ represents the six-column eigenvectors ${ }^{18}$ of the Hamiltonian at the same energy $E$, and parallel momentum $\mathbf{k}_{\|}$and the wave vector $k_{z i}$ classifies the states of heavy and light holes ( $n_{b}$ is the number of bands). The canonical wave vectors $\mathbf{k}_{\|}=\left(k_{x}, k_{y}\right)$ are conserved, so that mathematically the problem is reduced to a one-dimensional problem. The eigenvectors $V_{i}$ should be normalized to unity to ensure that the coefficients $a_{i}^{(L, R)}$ are directly proportional to the probability current density of the particular channels, i.e., to transmission (reflection) coefficients. If the Hamiltonian is written as

$$
H=H_{n}^{(2)} k_{z}^{2}+H_{n}^{(1)} k_{z}+H_{n}^{(0)}
$$

the wave vectors $k_{z i}$ can be found from the augmented eigenvalue problem

$$
\left(\begin{array}{cc}
\mathbf{0} & I \\
-\left(H_{n}^{(2)}\right)^{-1} H_{n}^{\prime} & -\left(H_{n}^{(2)}\right)^{-1} H_{n}^{(1)}\left(\mathbf{k}_{\|}\right)
\end{array}\right) V_{a}=k_{z} V_{a},
$$

where $V_{a}=\left(V_{i}, k_{z i} V_{i}\right)^{T}$ and $H_{n}^{\prime}=H_{n}^{(0)}-E_{n} I$. This leads to the characteristic polynomial with 12 solutions, which correspond to waves traveling in both directions. This procedure has the advantage that one obtains both wave vectors $k_{z i}$ and eigenvectors $V_{i}$ at once. The coefficients $A_{n}^{+}$correspond to the eigenvalues traveling with positive group velocity, i.e., $j_{z}>0$ and $\operatorname{Im}\left\{k_{z}\right\}=0$, or decaying in the positive direction $\operatorname{Im}\left\{k_{z}\right\}>0$. The number of positive modes should be half the total number of modes, since the relation

$$
E(-\mathbf{k}, \uparrow)=E(+\mathbf{k}, \downarrow)
$$

still holds even in the presence of a magnetic field and/or localized moments. This is a consequence of the time invariance of the Hamiltonian

$$
[\hat{\theta}, \hat{H}]=0, \quad \hat{\theta} \Psi(\mathbf{r}, t)=\Psi^{\dagger}(\mathbf{r},-t) .
$$

A typical trilayer structure (Ga, Mn)As/GaAs/AlAs/GaAs/(Ga, Mn)As used in experiments is shown in Fig. 1. It consists of two (Ga,Mn)As layers separated by a thin layer made of AlAs. Two additional layers of GaAs are inserted to prevent diffusion of $\mathrm{Mn}$ into AlAs. Since there are six channels possible for tunneling, i.e., mixing of different kinds of holes may happen depending on the scattering potential (barrier), the transmissions and reflection coefficients should be normalized to the group velocity ${ }^{17}$ 


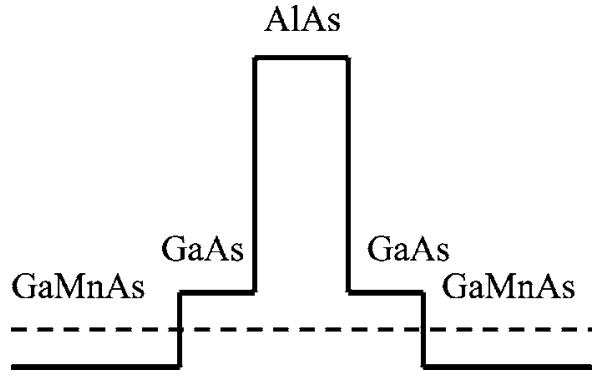

FIG. 1. Schematic band diagram of a trilayer structure made of two DMS layers $(\mathrm{Ga}, \mathrm{Mn})$ As separated by an AlAs layer acting as a barrier. Two additional layers of GaAs are inserted in between in order to prevent diffusion of $\mathrm{Mn}$ into the barrier.

$$
T_{\lambda \mu}=\left|\frac{A_{R \mu}^{+}}{A_{L \lambda}^{+}}\right| \frac{2}{v_{g r \mu}} .
$$

The quantities $A_{R \mu}^{+}$and $A_{L \lambda}^{+}$are amplitudes of the quantum mechanical waves right and left of the structure, while $\mu, \lambda$ denote various hole states. The conservation of the probability current leads to six relations among transmission and reflection coefficients

$$
\sum_{\mu}\left(T_{\lambda \mu}+R_{\lambda \mu}\right)=1
$$

Here the variable $\lambda$ assumes all six hole states $\left(h_{1}, h_{2}, l_{1}, l_{2}\right.$, $s_{1}$, and $s_{2}$ ). These relations are also useful to check the consistency of the numerical calculations. Formally present in Eq. (11) split-off band states $s_{1}, s_{2}$ do not contribute to the current since the band edge of the LH "upper subband" is still higher than the SO down subband $\left(\Delta_{S O}>\Delta_{e x}\right)$. They influence the current values, however, in an indirect way since the imaginary parts of $k_{s o}$ are small, and because the matrix component $\sqrt{2} Q$ (that contains $\hat{k}_{z}$ ) mixes LH and SO holes. Thus, one should take into account more precisely the dispersion relation $\epsilon_{h h, l h}(k)$ obtained from $H_{6 \times 6}$ when $\epsilon_{h h}$ approaches the SO band. A better insight into band alignment of materials is given in Fig. 2, with GaAs spacers excluded. The scheme of dispersions $E\left(k_{z}\right)$ are given for $k_{x}=0, k_{y}=0$, for $\left(\mathrm{Ga}_{0.96}, \mathrm{Mn}_{0.04}\right)$ As (first FM layer) and for pure AlAs and $\left(\mathrm{Ga}_{0.966}, \mathrm{Mn}_{0.034}\right)$ As (second FM layer). The valence band of AlAs is shifted by $0.75 \mathrm{eV}$ to the total barrier height AlAs/GaAs and GaAs/(Ga,Mn)As. The smaller splitting between the heavy holes as compared to the one of the light holes is a result of spin-orbit interaction ${ }^{19,20}$ and the fact that the magnetization $M$ is in plane.

\section{RESULTS}

The numerical method is based on the transfer matrix method for stratisfied media. The coefficients $A$ on either side are connected by

$$
\left(\begin{array}{c}
A^{+} \\
0
\end{array}\right)=T\left(\begin{array}{c}
I_{\lambda} \\
r
\end{array}\right),
$$

where the column $I_{\lambda}$ contains 1 for the $\lambda$ th channel, the other being zero, i.e., $I_{\lambda}=\delta_{i \lambda}$, while the $r$ subcolumn contains re-

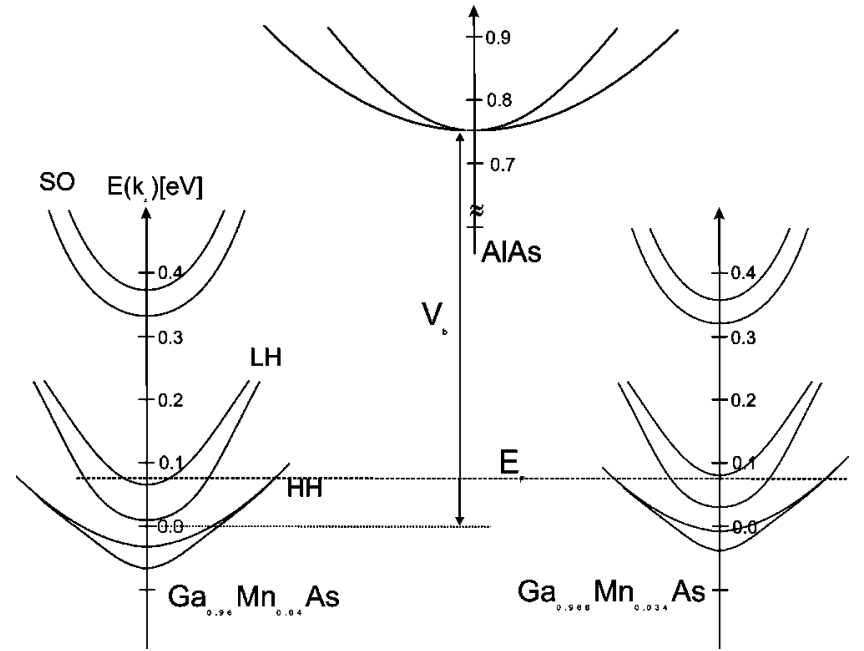

FIG. 2. Scheme of band positions of the two $(\mathrm{Ga}, \mathrm{Mn})$ As layers and the barrier of AlAs. The band diagram of GaAs and split-off band of AlAs are not shown for clarity.

flection coefficients. The matrix $M$ is composed of the boundary matching matrix $M_{i}$ and the propagation matrix $P$,

$$
T=M_{N}^{-1}\left(M_{N-1} P_{N} M_{N-1}^{-1}\right) \cdots\left(M_{1} P M_{1}^{-1}\right) M_{0},
$$

whose explicit expressions can be found elsewhere. ${ }^{21}$ In the case of a trilayer one has three layers GaAs/AlAs/GaAs, while two (thick) layers of (Ga,Mn)As act as the emitter and collector leads. It is found ${ }^{22}$ that GaAs acts as a barrier to (Ga,Mn)As, whose height depends on the manganese content. Its experimental value is in the range $(88,146) \mathrm{meV}$ for $x(\mathrm{Mn})(1.2-2.5 \%)$ according to Ref. 22 and consistent with Ref. 7. Having found transmission coefficients $T_{\lambda \mu}, \lambda, \mu$ $=1, \ldots, 6$ from Eq. (13), the current density is given by

$$
J_{C}=\frac{e}{8 \pi^{3} \hbar} \int_{0}^{k_{t \max }} k_{t} d k_{t} \int_{0}^{2 \pi} d \phi\left(\sum_{\mu \lambda} T_{\mu \lambda}\right),
$$

in the limit of small $V_{\text {bias }} \ll \epsilon_{F}$. The tunneling current, Eq. (14), is calculated for both alignment of the two ( $\mathrm{Ga}, \mathrm{Mn}) \mathrm{As}$, so that the tunneling magnetoresistance is found from

$$
T M R=\frac{J_{C p}-J_{C a}}{J_{C a}},
$$

where $J_{C p}$ is the density of tunneling current when the two FM films are parallel, while $J_{C a}$ denotes the current density when the two are antiparallel. For our numerical calculations we used the sample parameters of Ref. 5. These parameters are as follows: the width of two GaAs layers is $D_{0}=1 \mathrm{~nm}$, the barrier thickness $D$ of AlAs varied from $1.2 \mathrm{~nm}$ to $2.2 \mathrm{~nm}$, and the Mn molar contents of the two DMS layers are $4.0 \%$ and $3.3 \%$. The exchange energies corresponding to these values are $\Delta_{e x}^{L}=0.12 \mathrm{eV}$ and $\Delta_{e x}^{R}$ $=0.1 \mathrm{eV}$, respectively, assuming full alignment of manganese localized moments. The different manganese concentration is made deliberately in order to build in the discrepancy in coercivity of the two DMS layers, i.e., to allow an easy change from parallel to antiparallel orientation. The applied bias was $e V_{\text {bias }}=1 \mathrm{meV}$, much smaller than the Fermi level 


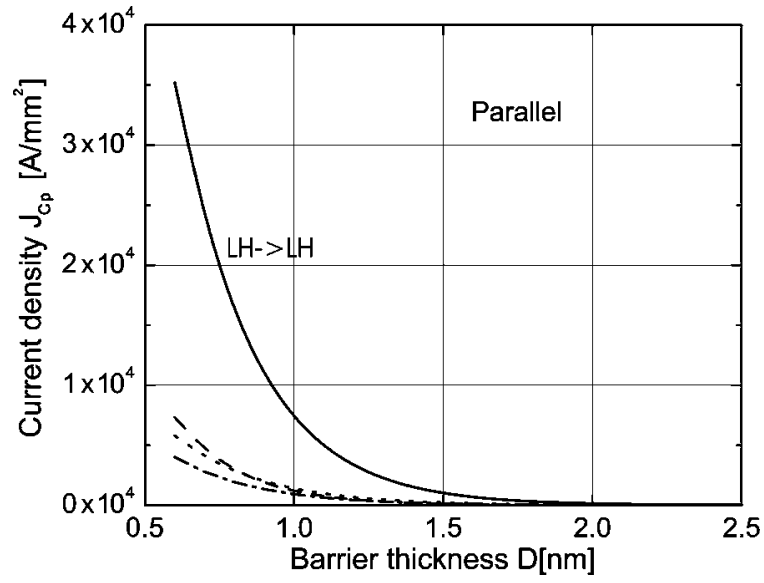

FIG. 3. Components of the current density $J_{C p}$ as a function of barrier thickness $D$ when the DMS layers are aligned parallel. Light hole to light hole transmission is dominant.

$\epsilon_{F} \approx 80 \mathrm{meV}$. The barrier height $V_{b}=0.75 \mathrm{eV}$ and the measurement was performed at low temperature $T=8 \mathrm{~K}$, which justifies using Eq. (14). The barrier height $V_{b 2}$ between $(\mathrm{Ga}, \mathrm{Mn}) \mathrm{As}$ and GaAs is taken to be approximately $0.2 \mathrm{eV}$, since $x(\mathrm{Mn})$ is higher than 3\%. The Luttinger parameters are $\gamma_{1}^{(1)}=6.85, \gamma_{2}^{(1)}=2.1, \gamma_{3}^{(1)}=2.9(\mathrm{GaAs})$, and $\gamma_{1}^{(2)}=3.45, \gamma_{2}^{(2)}$ $=1.29, \gamma_{3}^{(2)}=0.68$ (AlAs). In order to gain better insight in the process of tunneling, we show the current density for the case of parallel orientation of the two DMS layers (Fig. 3) and for antiparallel (Fig. 4) orientation. Out of the 36 possible transmissions, only 12 are nonzero since the split-off band is below the Fermi energy, and within the barrier there is no magnetic field. This means, for instance, that the transmissions between $\mathrm{HH} 1$ and $\mathrm{HH} 2$, and between LH1 and $\mathrm{LH} 2$, are not possible because of the presence of zeros on the small off-diagonal in the matrix elements of Eq. (2). However, out of these 12 channels only about four to eight are significant depending on the alignment of the ferromagnetic layers. The current components, when the two FM are parallel, are shown in Fig. 3. Only one component, light hole to light hole, is significantly larger than the other as a result of

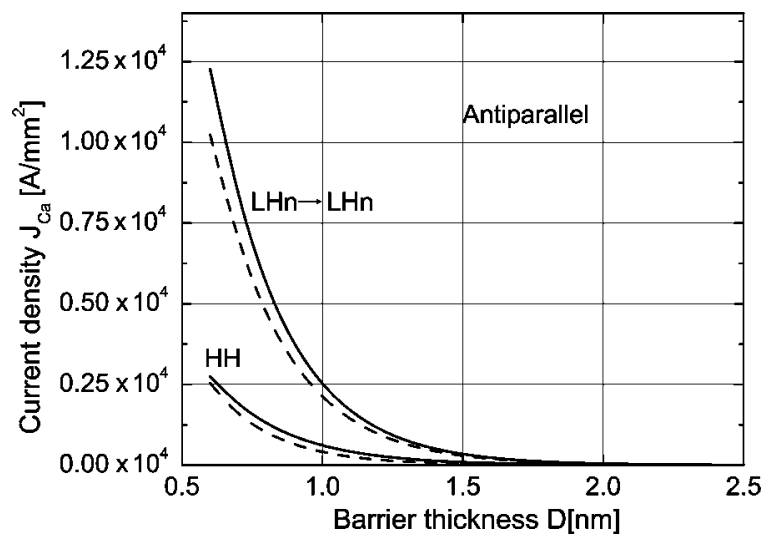

FIG. 4. Components of the current density $J_{C a}$ as a function of barrier thickness $D$ when the DMS layers are aligned antiparallel. Heavy to heavy hole component makes up less than $20 \%$ of the total current.

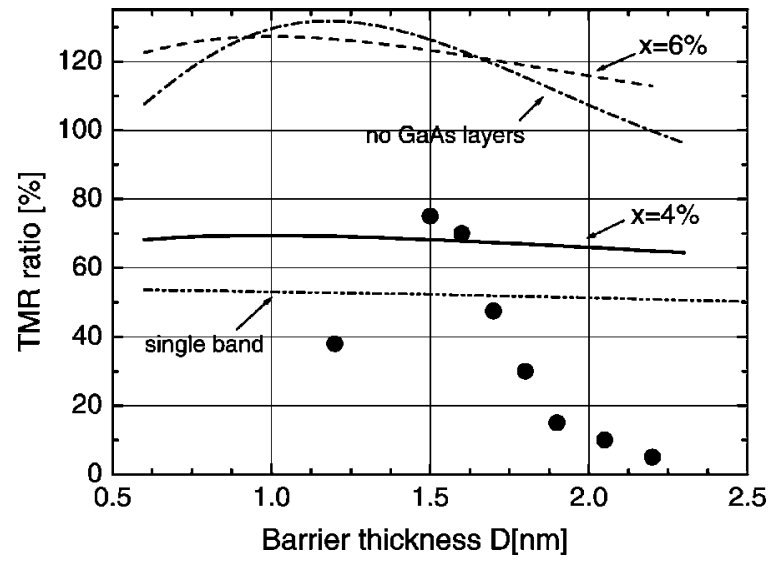

FIG. 5. Tunneling magnetoresistance ratio (TMR) as a function of the thickness $D$ of the AlAs tunnel barrier of AlAs (solid line) is shown along with experimental data taken from Ref. 5 (closed circles). For comparison, theoretical TMR without the two GaAs spacers (dash-dotted line), and within the single band approximation (dash-dot-dot) are given, all for $x(\mathrm{Mn})=(4.0,3.3) \%$. In addition, we present the result for a higher Mn content $x=(6.0,5.5) \%$ of the two layers.

their lighter mass. They exhibit rapid decay with the barrier thickness. In the next figure, i.e., Fig. 4, we present the results for the antiparallel case, where one can notice significantly smaller values for the tunnel currents. The closely spaced curves, two by two, are a consequence of a different Mn content of the two FM layers, i.e., they have different exchange splitting. Thus the transmission probabilities LH1 into LH1 and LH2 into LH2 are not the same. On the other hand, the different polarizations of heavy and light holes is because the $\mathrm{HH}$ eigenfunctions have a definite value of spin,

$$
\left|\frac{3}{2}, \frac{3}{2}\right\rangle=\frac{1}{\sqrt{2}}|X+i Y\rangle \uparrow ; \quad\left|\frac{3}{2},-\frac{3}{2}\right\rangle=\frac{i}{\sqrt{2}}|X-i Y\rangle \downarrow,
$$

while the LH eigenfunctions are composed of different spin subspaces,

$$
\begin{aligned}
& \left|\frac{3}{2}, \frac{1}{2}\right\rangle=\frac{i}{\sqrt{6}}[|X+i Y\rangle \downarrow-2|Z\rangle \uparrow], \\
& \left|\frac{3}{2},-\frac{1}{2}\right\rangle=\frac{1}{\sqrt{6}}[|X-i Y\rangle \downarrow+2|Z\rangle \downarrow] .
\end{aligned}
$$

Figure 5 shows the theoretical estimate for TMR (solid line), along with the experimental data from Ref. 5 (closed circles) for manganese concentration $x=4 \%$ (first FM layer) and $x=3.3 \%$ (second FM layer). A decent agreement is achieved only for two experimental points, while for higher $D$, the experimental data show a rapid decay. There could be several reasons for this discrepancy: the possibility of indirect tunneling, ${ }^{7}$ the presence of defects that spoil resonant tunneling, the presence of $\mathrm{Mn}$ in the barrier, etc. Despite the fact that the structure is made by the sophisticated molecular beam epitaxy (MBE) growth technique, the presence of As antisites, and interstitial $\mathrm{Mn}$ is inevitable in the fabrication of 
$(\mathrm{Ga}, \mathrm{Mn})$ As. Another reason is the presence of two GaAs layers on both sides, whose thickness is comparable with the width of the trilayer structure and is not changed in the experiment, while the barrier they impose is not so high. This is clearly shown on the same figure, where the dash-dotted line corresponds to the TMR in the absence of the two GaAs layers. The slope of the curve is more pronounced than in the previous case. On the other hand, the considered model does not have any fitting parameter, so that the semiquantitative agreement is satisfactory. Clearly, in order to enhance TMR, one should enlarge the spin-splitting of the holes in (Ga,Mn)As, e.g., by increasing the Mn content. Calculations of TMR for $x=6 \%(x=5.5 \%)$ are given in the same Fig. 5 (dashed line). The predicted value of the magnetoresistance for this $x(\mathrm{Mn})$ reaches $130 \%$, a promising value, but it was obtained under the assumption that there are no selfcompensation effects, either for the hole density or the magnetization of the layers.

\section{SINGLE BAND APPROXIMATION}

In order to gain better insight into the tunneling processes, we depart from the $6 \times 6$ Luttinger-Kohn model and analyze the problem within the single band approximation. This serves only as an illustration and to explain qualitatively the slow variation of the TMR ratio on the barrier thickness. The wave function in the three regions, for an incoming spin-up state is

$$
\begin{gathered}
\psi_{I}(z \leqslant 0)=V_{\uparrow} e^{i k_{z \uparrow} z}+r_{\uparrow \uparrow} V_{\uparrow} e^{-i k_{z \uparrow} z}+r_{\uparrow \downarrow} V_{\downarrow} e^{-i k_{z \downarrow} z} \\
\psi_{I I I}(0<z<d)=A_{\uparrow} V_{\uparrow} e^{i k_{z 2 \uparrow} z}+B_{\uparrow} V_{\uparrow} e^{-i k_{z 2 \uparrow} z}+A_{\downarrow} V_{\downarrow} e^{i k_{z 2 \uparrow} z} \\
+B_{\downarrow} V_{\downarrow} e^{-i k_{z 2 \downarrow} z} \\
\psi_{I I I}(z \geqslant d)=t_{\uparrow \uparrow} V_{\uparrow} e^{i k_{z \uparrow} z}+t_{\uparrow \downarrow} V_{\downarrow} e^{i k_{z \downarrow} z}
\end{gathered}
$$

where

$$
V_{\uparrow}=\frac{1}{\sqrt{2}}\left(\begin{array}{l}
1 \\
1
\end{array}\right), \quad V_{\downarrow}=\frac{1}{\sqrt{2}}\left(\begin{array}{c}
1 \\
-1
\end{array}\right) .
$$

To simplify the problem further, we neglect the influence of the two GaAs layers. The result for the TMER ratio is shown in Fig. 5 (dash-dot-dot line). The exchange energy was taken smaller $\left(\Delta_{e x 1} \approx 1 / 2 \Delta_{e x}\right)$ than in the full six-band treatment to account for the influence of spin-orbit interaction (see Fig. 2). Note that, within the single band approximation, the $\mathrm{HH}$ subbands are significantly split. ${ }^{10}$ However, this is only an approximation, since the spin-orbit coupling tends to decrease the heavy hole splitting. Nevertheless, the HH currents for both orientations are significantly smaller than that of the light holes since the heavy holes tunnels less efficient as they have heavier mass. In Fig. 6 we show the current components within the single band approximation when the ferromagnetic layers are parallel (without GaAs). As in the case of the $6 \times 6 \mathrm{LK}$, the current for $\mathrm{LH} \rightarrow \mathrm{LH}$ is larger than

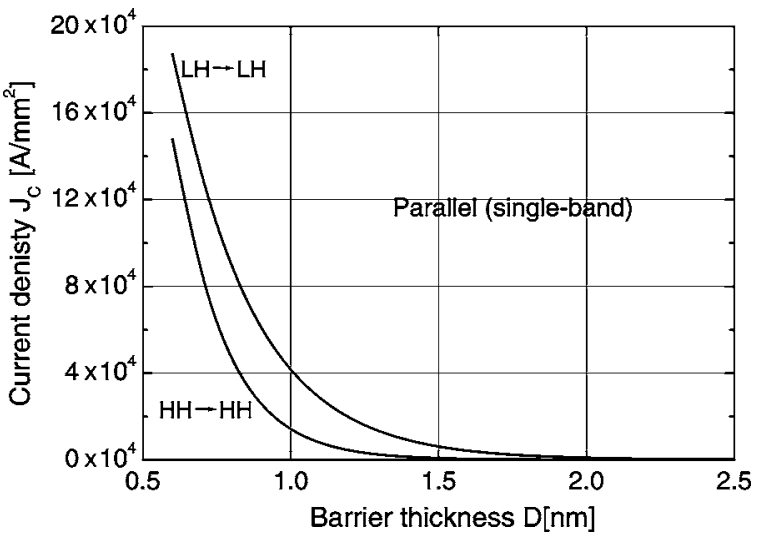

FIG. 6. Light and heavy hole components of the tunneling current, within the single band approximation, when the FM layers are parallel.

the $\mathrm{HH} \rightarrow \mathrm{HH}$ current. In this case the difference is smaller because we neglected the role of the two GaAs layers acting as a low barrier $V_{b 2}=0.2 \mathrm{eV}$, and the fact that the effective mass of holes does not change with $\mathbf{k}_{\|}$. One can take into account their influence by adding the effective width $D_{\text {eff }}-D=0.5 \mathrm{~nm}$, and then values of the current will be comparable with those given in Fig. 3.

\section{CONCLUSIONS}

We have investigated spin-dependent tunneling through a diluted magnetic semiconductor trilayer structure. The problem is investigated within the $6 \times 6$ Luttinger-Kohn model for the valence bands, and it was assumed that the TMR is due to direct tunneling because of the high quality of the semiconductor layers. However, the presence of interstitial manganese atoms and the low temperature MBE that introduces As antisites spoil the coherence of the hole wave functions, so that only a semiquantative agreement with experiment is achieved. Our theoretical results should be considered as an upper bound, and the main discrepancy between the model and the experimental data is that our predictions of the TMR as a function of the barrier thickness do not show a rapid decay with the barrier thickness. Possible reasons could be noncoherent tunneling as a result of the presence of defect As antisites, interstitial Mn, etc. Previous theoretical predictions that $\mathrm{HH}$ spin-splitting is reduced by spin-orbit interaction is confirmed by our results. However, their influence on the total TMR is smaller than that of the light holes because their tunneling currents (for both orientations) are smaller due to their heavier mass in the tunneling direction.

\section{ACKNOWLEDGMENTS}

This work was supported by the Flemish Science Foundation (FWO-Vl) and the Belgian Science Policy. 
*Electronic address: predrag.krstajic@ua.ac.be

'Electronic address: francois.peeters@ua.ac.be

${ }^{1}$ S. A. Wolf, D. D. Awschalom, R. A. Buhrman, J. M. Daughton, S. von Molnar, M. L. Roukes, A. Y. Chtchelkanova, and D. M. Treger, Science 294, 1488 (2001).

${ }^{2}$ S. Datta and B. Das, Appl. Phys. Lett. 56, 665 (1990).

${ }^{3}$ T. Dietl, Semicond. Sci. Technol. 17, 377 (2002).

${ }^{4}$ D. Chiba, F. Matsukura, and H. Ohno, Physica E (Amsterdam) 21, 966 (2004).

${ }^{5}$ M. Tanaka and Y. Higo, Phys. Rev. Lett. 87, 026602 (2001).

${ }^{6}$ Y. Higo, H. Shimizu, and M. Tanaka, J. Appl. Phys. 89, 6745 (2001)

${ }^{7}$ R. Mattana, M. Elsen, J.-M. George, H. Jaffrès, F. N. Van Dau, A. Fert, M. F. Wyczisk, J. Olivier, P. Galtier, B. Lépine, A. Guivarc'h, and G. Jézéquel, Phys. Rev. B 71, 075206 (2005).

${ }^{8}$ S. S. Makler, M. A. Boselli, J. Weberszpil, X. F. Wanga, and I. C. da Cunha Lima, Physica B 320, 396 (2002); S. S. Makler, M. A. Boselli, J. Weberszpil, X. F. Wanga, and I. C. da Cunha Lima, ibid. 354, 348 (2004).

${ }^{9}$ J. M. Luttinger and W. Kohn, Phys. Rev. 97, 869 (1955).

${ }^{10}$ A. G. Petukhov, A. N. Chantis, and D. O. Demchenko, Phys. Rev.
Lett. 89, 107205 (2002).

${ }^{11}$ A. G. Petukhov, D. O. Demchenko, and A. N. Chantis, Phys. Rev. B 68, 125332 (2003).

${ }^{12}$ T. Dietl, H. Ohno, and F. Matsukura, Phys. Rev. B 63, 195205 (2001).

${ }^{13}$ S. C. P. Rodrigues, L. M. R. Scolfaro, J. R. Leite, I. C. da Cunha Lima, G. M. Sipahi, and M. A. Boselli, Phys. Rev. B 70, 165308 (2004).

${ }^{14}$ R. Wessel and M. Altarelli, Phys. Rev. B 39, 12802 (1989).

${ }^{15}$ E. T. Yu, M. K. Jackson, and T. C. McGill, Appl. Phys. Lett. 55, 744 (1989).

${ }^{16}$ N. Malkova and U. Ekenberg, Phys. Rev. B 66, 155324 (2002).

${ }^{17}$ P. Krstajić and F. M. Peeters, Phys. Rev. B 71, 115321 (2005).

${ }^{18}$ L. C. Andreani, A. Pasquarello, and F. Bassani, Phys. Rev. B 36, 5887 (1987).

${ }^{19}$ T. Dietl, A. Haury, and Y. M. d'Aubigne, Phys. Rev. B 55, R3347 (1997).

${ }^{20}$ T. Dietl, J. Phys.: Condens. Matter 16, S5471 (2004).

${ }^{21}$ C. Y. Chao and S. L. Chuang, Phys. Rev. B 43, 7027 (1991).

${ }^{22}$ Y. Ohno, I. Arata, F. Matsukura, and H. Ohno, Physica E (Amsterdam) 13, 521 (2002). 